\title{
Cross-Phase Modulation in Multispan WDM Optical Fiber Systems
}

\author{
Rongqing Hui, Senior Member, IEEE, Kenneth R. Demarest, Senior Member, IEEE, \\ and Christopher T. Allen, Senior Member, IEEE
}

\begin{abstract}
The spectral characteristics of cross-phase modulation (XPM) in multispan intensity-modulation direct-detection (IM-DD) optical systems are investigated, both experimentally and theoretically. XPM crosstalk levels and its spectral features are found to be strongly dependent on fiber dispersion and optical signal channel spacing. Interference between XPMinduced crosstalk effects created in different amplified fiber spans is also found to be important to determine the overall frequency response of XPM crosstalk effects. XPM crosstalk between channels with different data rates is evaluated. The crosstalk level between higher and lower bit rate channels is found to be similar to that between two lower bit rate channels. The effect of dispersion compensation on XPM crosstalk in multispan optical systems is discussed and per span dispersion compensation was found to be the most effective way to minimize the effect of XPM crosstalk.
\end{abstract}

\section{INTRODUCTION}

C ROSS-PHASE modulation (XPM) has an important impact on the performance of high-speed wavelength division multiplexing (WDM) optical fiber communication systems [1], [2]. XPM originates from the Kerr effect in optical fibers, where intensity modulation of one optical carrier can modulate the phases of other copropagating optical signals in the same fiber. Unlike coherent optical systems, intensitymodulation direct-detection (IM-DD) optical systems are not particularly sensitive to signal-phase fluctuations. Therefore, crosstalk-induced phase modulation alone is not a direct source of performance degradation in IM-DD systems. However, due to the chromatic dispersion of optical fibers, phase modulation can be converted into intensity modulation [3] and, thus, can degrade the IM-DD system performance.

It has been reported previously that XPM created phasemodulation is inversely proportional to the signal baseband modulation frequency [4]. Since only the phase modulation was analyzed in [4], the results are not directly applicable to IM-DD optical systems. On the other hand, the effect of phase-noise to intensity-noise conversion through fiber dispersion was also studied [3] for single channel optical systems where the phase noise is originated from semiconductor lasers. In WDM-IM-DD optical systems, XPM-induced crosstalk involves both phase-modulation generation through

Manuscript received July 6, 1998; revised March 15, 1999. This work was supported in part by Sprint Corporation and the NSF under Grant EPSCoR/ECS 963-2617.

The authors are with the Information and Telecommunication Technology Center, the Department of Electrical Engineering and Computer Science, The University of Kansas, Lawrence, KS 66045 USA.

Publisher Item Identifier S 0733-8724(99)04516-8. the nonlinear Kerr effect and phase-noise to intensity-noise conversion through fiber dispersion.

In this paper, we report the results of an experimental and theoretical study on the frequency response of XPMinduced crosstalk in multispan WDM optical systems, both with a single fiber type and with mixed fiber configurations. Section II presents the theoretical analysis, where the crosstalk level is found to be dependent on the both optical channel spacing and the fiber dispersion. Interference between XPMinduced crosstalk effects created in different amplified optical spans is found to have a strong impact on the overall spectral feature of XPM-induced crosstalk. A simple analytical expression is obtained to describe the XPM-induced crosstalk. Section III details the experimental procedure, major results and the comparison with theory. The impact of XPM crosstalk on the system performance will be discussed in Section IV. In particular, XPM crosstalk between channels with different data rates is evaluated in hybrid WDM optical systems. The crosstalk level between high and low bit rate channels is found to be similar to that between two low bit rate channels. The effect of dispersion compensation on XPM crosstalk in multispan optical systems is also discussed, and per span dispersion compensation is found to be the most effective way to minimize the impact of XPM crosstalk.

\section{THEORETICAL ANALYSIS}

The theoretical analysis begins with the nonlinear wave propagation equation [5]. Consider probe and pump optical signals, $A_{j}(t, z)$ and $A_{k}(t, z)$, copropagating in the same optical fiber. The evolution of the probe wave is described by (a similar equation can be written for the pump wave)

$$
\begin{aligned}
\frac{\partial A_{j}(t, z)}{\partial z}= & -\frac{\alpha}{2} A_{j}(t, z)-\frac{1}{v_{j}} \frac{\partial A_{j}(t, z)}{\partial t} \\
& -\frac{i \beta_{2}}{2} \frac{\partial^{2} A_{j}(t, z)}{\partial t^{2}} \\
& +i \gamma_{j} p_{j}(t, z) A_{j}(t, z) \\
& +i \gamma_{j} 2 p_{k}\left(t-z / v_{k}, z\right) A_{j}(t, z)
\end{aligned}
$$

where $\alpha$ is the attenuation coefficient of the fiber, $\beta_{2}$ is the fiber chromatic dispersion parameter, $\gamma_{j}=2 \pi n_{2} /\left(\lambda_{j} A_{\text {eff }}\right)$ is the nonlinear coefficient, $n_{2}$ is the nonlinear refractive index, $\lambda_{j}$ and $\lambda_{k}$ are the probe and the pump signal wavelengths, $A_{\text {eff }}$ is the fiber effective core area, $p_{k}=\left|A_{k}\right|^{2}$ and $p_{j}=\left|A_{j}\right|^{2}$ are optical powers of the pump and the probe, respectively. Note that the last term in the right-hand-side (RHS) of (1) is slightly 
different from that in the literature (e.g., [5, eq. (7.1.17)]). Due to chromatic dispersion, the pump and the probe waves generally travel at different speeds and this difference must be taken into account in the calculation of XPM because it introduces the walk-off between the two waves. We use $p_{j}$ and $v_{k}$ to represent the group velocities of the two channels.

On the RHS of (1), the first term is attenuation, the second term is linear phase delay, the third term accounts for chromatic dispersion, the fourth term is responsible for self-phase modulation (SPM) and the fifth term is the XPM in the probe signal $(j)$, induced by the pump signal $(k)$. The strength of the XPM is proportional to the optical power in the pump and the fiber nonlinearity. In order to simplify the analysis and focus our attention on the effect of XPM induced interchannel crosstalk, we neglect the interaction between SPM and XPM and pretend that these two act independently. We will assume that the probe signal is operated in continuous wave (CW), whereas the pump signal is modulated with a sinusoidal wave at a frequency $\Omega$. Although the effect of SPM for both the probe and the pump channels are neglected in this XPM calculation, a complete system performance evaluation must take into account the effect of SPM and other nonlinear effects separately. This approximation is valid as long as the pump signal waveform is not appreciably changed by the SPMinduced distortion before its optical power is significantly reduced by the fiber attenuation. Under this approximation, we neglect the fourth term on the right-hand side (RHS) of (1) in our XPM evaluation. Using the substitutions $T=t-z / v_{j}$ and $A_{j}(t, z)=E_{j}(T, z) \exp (-\alpha z / 2)$, we have,

$$
\begin{aligned}
\frac{\partial E_{j}(T, z)}{\partial z}= & -\frac{i \beta_{2}}{2} \frac{\partial^{2} E_{j}(T, z)}{\partial T^{2}}+i \gamma_{j} 2 p_{k}\left(T-d_{j k} z, 0\right) \\
& \cdot \exp (-\alpha z) E_{j}(T, z)
\end{aligned}
$$

where $d_{j k} \equiv\left(1 / v_{j}\right)-\left(1 / v_{k}\right)$ is the relative walk-off between the probe and the pump. Using a linear approximation, the walk-off $d_{j k}$ can be expressed as $d_{j k}=D \Delta \lambda_{j k}$, where $D=-\left(2 \pi c / \lambda^{2}\right) \beta_{2}$ is the fiber dispersion coefficient, $\Delta \lambda_{j k}$ and $\lambda$ are, the wavelength spacing and the average wavelength between the probe and the pump, respectively, and $c$ is the light velocity. Here a linear approximation is used for $d_{j k}$ for simplicity and higher order dispersion effects are neglected. This is valid when the channel spacing $\Delta \lambda_{j k}$ is not too large. Coincidentally, this is the condition where cross-phase modulation is significant.

In general, dispersion and nonlinearity act together along the fiber. However, in an infinitesimal fiber section $d z$, we can assume that the dispersive and the nonlinear effects act independently, the same idea as used in the split-step Fourier method [5]. Let $E_{j}(T, z)=\left|E_{j}\right| \exp \left[i \phi_{j}(T, z)\right]$, where $\left|E_{j}\right|$ and $\phi_{j}$ are the amplitude and the phase of the optical field, respectively, of the probe channel. Taking into account the effect of cross-phase modulation alone, at $z=z^{\prime}$, the nonlinear phase modulation in the probe signal induced by the pump signal in the small fiber section $d z$ can be obtained as

$$
d \phi_{j}\left(T, z^{\prime}\right)=\gamma_{j} 2 p_{k}\left(T-d_{j k} z^{\prime}, 0\right) \exp \left(-\alpha z^{\prime}\right) d z
$$

The Fourier transformation of this phase variation gives

$$
d \tilde{\phi}_{j}\left(\Omega, z^{\prime}\right)=2 \gamma_{j} p_{k}(\Omega, 0) e^{\left(-\alpha+i \Omega d_{j k}\right) z^{\prime}} d z
$$

Neglecting the intensity fluctuation of the probe channel, this phase change corresponds to a change in the electrical field, $\bar{E}_{j} \exp \left[i d \phi_{j}\left(T, z^{\prime}\right)\right] \approx \bar{E}_{j}\left[1+i d \phi_{j}\left(T, z^{\prime}\right)\right]$, or, in the Fourier domain, $\bar{E}_{j}\left[1+i d \tilde{\phi}_{j}\left(\Omega, z^{\prime}\right)\right]$, where $d \tilde{\phi}_{j}\left(\Omega, z^{\prime}\right)$ is the Fourier transform of $d \phi_{j}\left(T, z^{\prime}\right)$ and $\bar{E}_{j}$ represents the average field amplitude.

Due to chromatic dispersion of the fiber, this phase variation generated at $z=z^{\prime}$ is converted into an amplitude variation at the end of the fiber $z=L$. Taking into account only the dispersion and a source term of the phase perturbation at $z=z^{\prime}$, the Fourier transform of (2) becomes

$\frac{\partial \tilde{E}_{j}(\Omega, z)}{d z}=\frac{i \beta_{2} \Omega^{2}}{2} \cdot \tilde{E}_{j}(\Omega, z)+\bar{E}_{j}\left[1+i d \tilde{\phi}\left(\Omega, z^{\prime}\right)\right] \delta\left(z-z^{\prime}\right)$

where the Kronecker delta $\delta\left(z-z^{\prime}\right)$ is introduced to take into account the fact that source term exists only in an infinitesimal fiber section at $z=z^{\prime}$. Therefore, at the fiber output $z=L$, the probe field is

$$
\tilde{E}_{j}(\Omega, L)=\bar{E}_{j}+i d \tilde{\phi}_{j}\left(\Omega, z^{\prime}\right) \bar{E}_{j} \exp \left[i \beta_{2} \Omega^{2}\left(L-z^{\prime}\right) / 2\right] .
$$

The optical power variation caused by the nonlinear phase modulation created in the short section $d z$ at $z=z^{\prime}$ is thus

$$
\begin{aligned}
\Delta \tilde{a}_{j k}\left(\Omega, z^{\prime}, L\right) & =\left|\tilde{E}_{j}(\Omega, L)\right|^{2}-\bar{E}_{j}^{2} \\
& =-2 \bar{E}_{j}^{2} d \tilde{\phi}_{j}\left(\Omega, z^{\prime}\right) \sin \left[\beta_{2} \Omega^{2}\left(L-z^{\prime}\right) / 2\right]
\end{aligned}
$$

where a linearization has been made considering that $d \tilde{\phi}_{j}$ is infinitesimal.

Using $E_{j}(T, z)=A_{j}\left(T+z / v_{j}, z\right) \exp (\alpha z / 2)$ and (3), integrating all cross-phase modulation contributions along the fiber, we obtain the total intensity fluctuation at the end of the fiber

$$
\begin{aligned}
\Delta \tilde{s}_{j k}(\Omega, L)= & -4 \gamma_{j} p_{j}(0) e^{-\left(\alpha-i \Omega / v_{j}\right) L} \int_{0}^{L} p_{k}(\Omega, 0) \\
& \cdot \sin \left[\beta_{2} \Omega^{2}\left(L-z^{\prime}\right) / 2\right] e^{-\left(\alpha-i \Omega d_{j k}\right) z^{\prime}} d z^{\prime}
\end{aligned}
$$

where, $p_{j}=\left|A_{j}\right|^{2}$ and $\Delta \tilde{s}_{j k}(\Omega, L)=\Delta \tilde{a}_{j k}(\Omega, L) e^{-\alpha L}$ represents the fluctuation of $A_{j}$. After integration, we have,

$$
\begin{aligned}
\Delta \tilde{s}_{j k}(\Omega, L) & \\
= & 2 p_{j}(L) \gamma_{j} e^{i \Omega / v_{j} L} p_{k}(\Omega, 0) \\
& \cdot\left\{\frac{\exp \left(i \beta_{2} \Omega^{2} L / 2\right)-\exp \left(-\alpha+i \Omega d_{j k}\right) L}{i\left(\alpha-i \Omega d_{j k}+i \beta_{2} \Omega^{2} / 2\right)}\right. \\
& \left.-\frac{\exp \left(-i \beta_{2} \Omega^{2} L / 2\right)-\exp \left(-\alpha+i \Omega d_{j k}\right) L}{i\left(\alpha-i \Omega d_{j k}-i \beta_{2} \Omega^{2} / 2\right)}\right\}
\end{aligned}
$$

where $p_{j}(0)$ and $p_{j}(L)$ are the probe optical power at the input and the output of the fiber, respectively. Under the assumptions that $\exp (-\alpha L) \ll 1$ and that the modulation bandwidth is much smaller than the channel spacing, i.e., $d_{j k} \gg \beta_{2} \Omega / 2$, we find a simpler frequency domain description 
of the intensity fluctuation in the probe channel caused by the intensity modulation of the pump channel

$$
\Delta \tilde{s}_{j k}(\Omega, L)=4 \gamma_{j} p_{j}(L) p_{k}(\Omega, 0) \frac{\sin \left(\beta_{2} \Omega^{2} L / 2\right)}{\alpha-i \Omega d_{j k}} e^{i \Omega / v_{j} L} .
$$

Equation (6) can be generalized to analyze multispan optically amplified systems, where the total intensity fluctuation at the receiver is the accumulation of XPM contributions created by each fiber span. For a system having a total of $N$ amplified fiber spans, the XPM created in the $m$ th span produces an intensity modulation $\Delta \tilde{s}_{j k}^{(m)}\left(\Omega, L_{N}\right)$ at the end of the system. Even though the phase modulation creation depends only on the pump power and the walk-off within the $m$ th span, the phase-to-intensity conversion depends on the accumulated dispersion of the fibers from the $m$ th to the $N$ th spans. Therefore, we have

$$
\begin{aligned}
\Delta \tilde{s}_{j k}^{(m)}\left(\Omega, L_{N}\right) & \\
= & 4 \gamma_{j} p_{j}\left(L_{N}\right) p_{k}^{(m)}(\Omega, 0) \exp \left[i \Omega \sum_{n=1}^{m-1} d_{j k}^{(n)} L^{(n)}\right] \\
& \quad \frac{\sin \left[\Omega^{2} \sum_{n=m}^{N} \beta_{2}^{(n)} L^{(n)} / 2\right]}{\alpha-i \Omega d_{j k}^{(i)}} \exp \left(i \Omega L_{N} / v_{j}\right)
\end{aligned}
$$

where, $L_{N}=\sum_{n=1}^{N} L^{(n)}$ is the total fiber length in the system, $L^{(m)}$ and $\beta_{2}^{(m)}$ are fiber length and dispersion of the $m$ th span [where $L^{(0)}=0$ ], $p_{k}^{(m)}(\Omega, 0)$ is the pump signal input power spectrum in the $m$ th span and $d_{j k}^{(m)}$ is the relative walk-off between two channels in the $m$ th span, [where $d_{j k}^{(0)}=0$ ]. To generalize $(6)$ to $(7)$, the term $\sin \left(\beta_{2} \Omega^{2} L / 2\right)$ in (6) is replaced by $\sin \left[\Omega^{2} \sum_{n=m}^{N} \beta_{2}^{(n)} L^{(n)} / 2\right]$ in (7) to take into account the linear accumulation of dispersion. Another important effect that must be taken into account, is that the pump and the probe waves travel at different speeds. The phase difference between the pump and the probe waves at the input of the $m$ th span is different from that at the input of the first span. The walk-off dependent term $\exp \left[i \Omega \sum_{n=1}^{m-1} d_{j k}^{(n)} L^{(n)}\right]$ in (7) takes into account the walk-off between the probe and the pump channels before they both enter into the $m$ th fiber span.

Finally, contributions from all fiber spans add up and therefore, the intensity fluctuation induced by the cross-phase modulation of the whole system can be expressed as

$$
\Delta \tilde{S}_{j k}\left(\Omega, L_{N}\right)=\sum_{m=1}^{N} \Delta \tilde{s}_{j}^{(m)}\left(\Omega, L_{N}\right) .
$$

In the time domain, the probe output optical power with $\mathrm{XPM}$-induced crosstalk is,

$$
p_{j k}\left(t, L_{N}\right)=p_{j}\left(L_{N}\right)+\Delta S_{j k}\left(t, L_{N}\right)
$$

where $\Delta S_{j k}\left(t, L_{N}\right)$ is the inverse Fourier transform of $\Delta \tilde{S}_{j k}\left(\Omega, L_{N}\right)$ and $p_{j}\left(L_{N}\right)$ is the probe output without XPM. $\Delta S_{j k}\left(t, L_{N}\right)$ has a zero mean. The originally $\mathrm{CW}$ probe is intensity modulated by the pump through the XPM process.
After the square-law detection of a photodiode, the electrical power spectral density is the Fourier transform of the autocorrelation of the time domain optical intensity waveform. Therefore, we have,

$$
\rho_{j}\left(\Omega, L_{N}\right)=\eta^{2}\left\{p_{j}^{2}\left(L_{N}\right) \delta(\Omega)+\left|\Delta \tilde{S}_{j k}\left(\Omega, L_{N}\right)\right|^{2}\right\}
$$

where $\delta$ is the Kronecker delta and $\eta$ is the photodiode responsivity. For $\Omega>0$, the XPM induced electrical power spectral density in the probe channel, normalized to its power level without this effect can be expressed as,

$$
\begin{aligned}
& \Delta p_{j k}\left(\Omega, L_{N}\right) \frac{\eta^{2}\left|\Delta \tilde{S}_{j k}\left(\Omega, L_{N}\right)\right|^{2}}{\eta^{2} p_{j}^{2}\left(L_{N}\right)} \\
&=\left|\sum_{i=1}^{N}\left\{\begin{array}{l}
4 \gamma_{j} p_{k}^{(i)}(\Omega, 0) \exp \left[i \Omega \sum_{n=1}^{i-1} d_{j k}^{(n)} L^{(n)}\right] \\
\sin \left[\Omega^{2} \sum_{n=i}^{N} \beta_{2}^{(n)} L^{(n)} / 2\right] \\
\alpha-i \Omega d_{j k}^{(i)}
\end{array}\right\}\right|^{2} .
\end{aligned}
$$

We define $\Delta p_{j k}\left(\Omega, L_{N}\right)$ as the normalized XPM power transfer function, which can be directly measured by a microwave network analyzer. It is worth mentioning here that in the derivation of (11), we have neglected the intensity fluctuation of the probe signal before it reaches the end of the system. This is indeed a small signal approximation, which is valid when the XPM-induced crosstalk is only a perturbation to the probe signal. The justification of the small signal approximation has been discussed in [8]. In fact, if this crosstalk level is less than, for example, $20 \%$ of the signal, the second-order effect caused by this small intensity fluctuation through SPM is considered to be negligible.

\section{EXPERIMENTS}

An experimental setup designed to measure the XPM frequency response is shown in Fig. 1. Two external-cavity tunable semiconductor lasers (ECL) emitting at $\lambda_{j}$ and $\lambda_{k}$, respectively, are used as sources for the probe and the pump signals. The probe signal is $\mathrm{CW}$ and the pump signal is externally modulated by the signal from a microwave network analyzer. The two optical signals are combined by a 3-dB coupler and then sent to an erbium-doped fiber amplifier (EDFA) to boost the optical power. A tunable optical filter is used at the receiver to select the probe signal and suppress the pump signal. After passing through an optical preamplifier, the probe signal is detected by a $32-\mathrm{GHz}$ bandwidth photo diode, amplified by a $10-\mathrm{GHz}$ bandwidth microwave amplifier, and then sent to the receiver port of the network analyzer.

Fig. 2 shows the normalized XPM frequency response measured at the output of a fiber link consisting of a single $114 \mathrm{~km}$ span of nonzero dispersion-shifted fiber (NZDSF). 


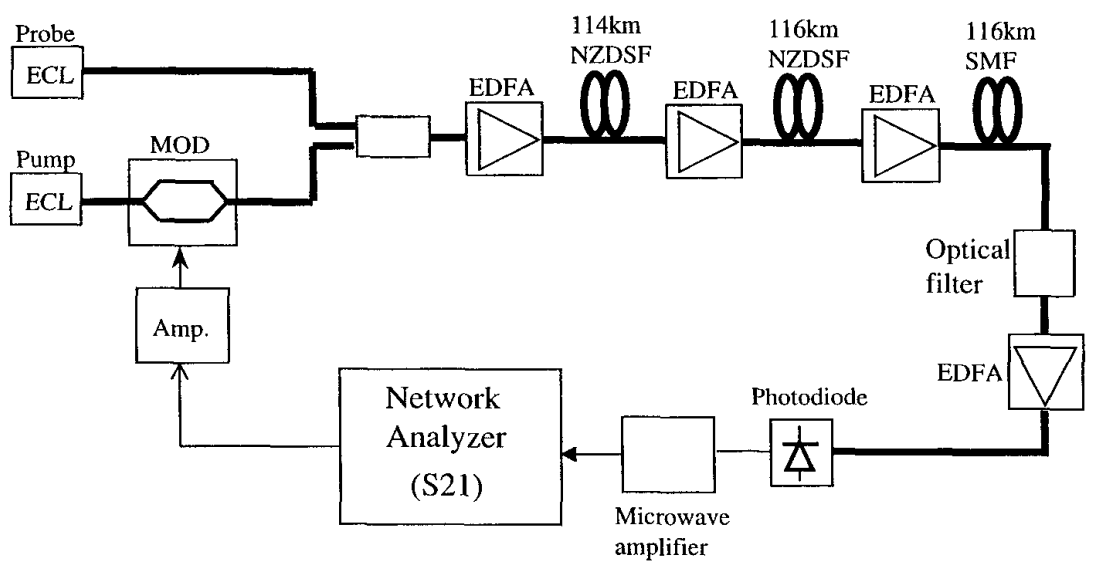

Fig. 1. Experimental setup. ECL: external cavity semiconductor laser. MOD: optical modulator.

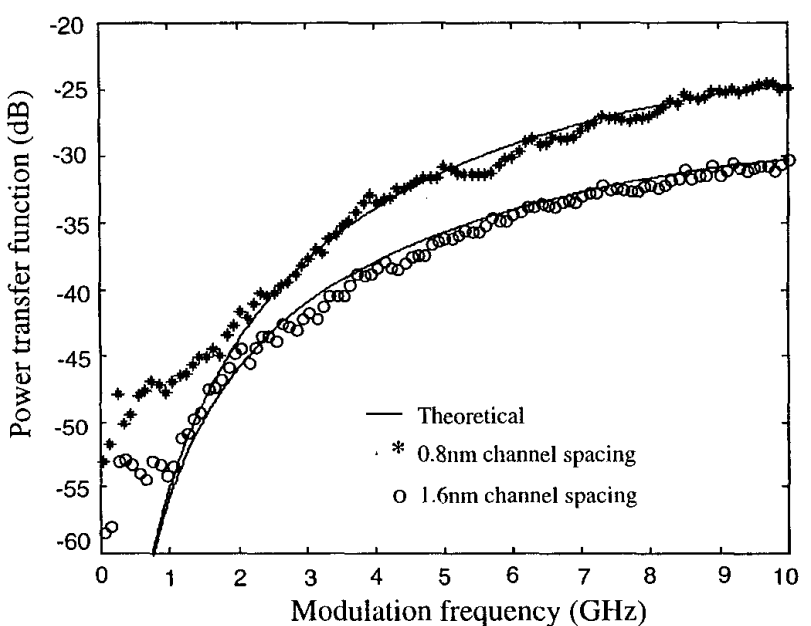

Fig. 2. XPM frequency response in the system with single span (114 $\mathrm{km})$ nonzero dispersion shifted fiber. Stars: $0.8 \mathrm{~nm}$ channel spacing $\left(\lambda_{\text {probe }}=1559 \mathrm{~nm}\right.$ and $\left.\lambda_{\text {pump }}=1559.8 \mathrm{~nm}\right)$, open circles: $1.6 \mathrm{~nm}$ channel spacing $\left(\lambda_{\text {probe }}=1559 \mathrm{~nm}\right.$ and $\left.\lambda_{\text {pump }}=1560.6 \mathrm{~nm}\right)$. Continuous lines are corresponding theoretical results.

The channel spacings used to obtain this figure were $0.8 \mathrm{~nm}$ $\left(\lambda_{j}=1559 \mathrm{~nm}, \lambda_{k}=1559.8 \mathrm{~nm}\right)$ and $1.6 \mathrm{~nm}\left(\lambda_{j}=1559 \mathrm{~nm}\right.$, $\left.\lambda_{k}=1560.6 \mathrm{~nm}\right)$. Corresponding theoretical results obtained from (11) are also plotted in the same figure. In order to have the best fit to the measured results, parameters used in the calculation were chosen to be $\lambda_{0}=1520.2 \mathrm{~nm}, S_{0}=0.075$ $\mathrm{ps} / \mathrm{km} / \mathrm{nm}^{2}, n_{2}=2.35 \cdot 10^{-20} \mathrm{~m}^{2} / \mathrm{W}, A_{\text {eff }}=5.5 \cdot 10^{-11} \mathrm{~m}^{2}$ and $\alpha=0.25 \mathrm{~dB} / \mathrm{km}$. These values agree with nominal parameter values of the NZDSF used in the experiment. Both the probe and the pump signal input optical powers were $11.5 \mathrm{dBm}$, and the pump channel modulation frequency was swept from 50 $\mathrm{MHz}$ to $10 \mathrm{GHz}$. In order to avoid significant higher order harmonics generated from the $\mathrm{LiNbO}_{3}$ Mach-Zehnder intensity modulator, the modulation index is chosen to be approximately $50 \%$. High-pass characteristics are clearly demonstrated in both curves in Fig. 2. This is qualitatively different from the frequency dependence of phase-modulation obtained in [4]. In that analysis, the conversion from phase-modulation to

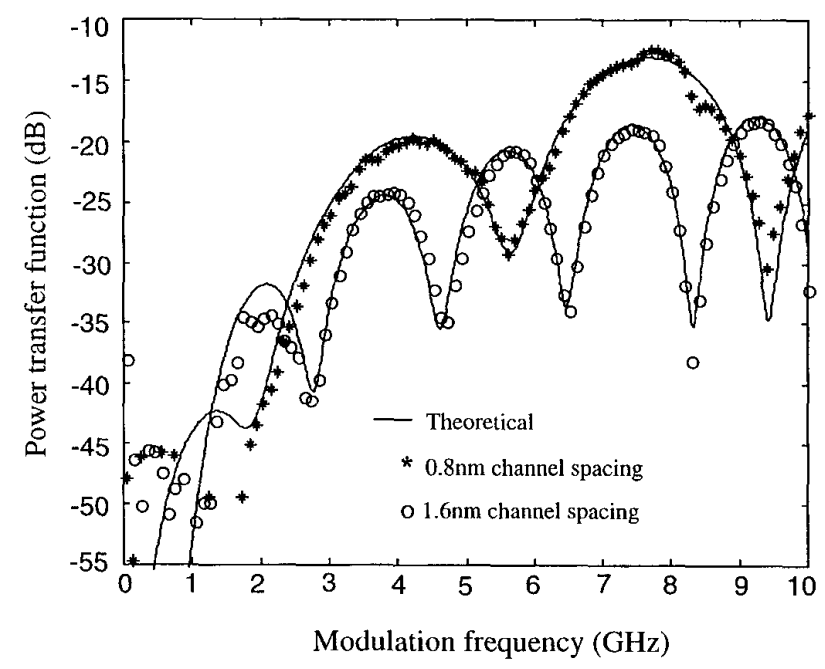

Fig. 3. XPM frequency response in the system with two spans (114 and $116 \mathrm{~km}$ ) of nonzero dispersion shifted fiber. Stars: $0.8 \mathrm{~nm}$ channel spacing $\left(\lambda_{\text {prabe }}=1559 \mathrm{~nm}\right.$ and $\left.\lambda_{\text {punp }}=1559.8 \mathrm{~nm}\right)$, open circles: $1.6 \mathrm{~nm}$ channel spacing $\left(\lambda_{\text {probe }}=1559 \mathrm{~nm}\right.$ and $\left.\lambda_{\text {pump }}=1560.6 \mathrm{~nm}\right)$. Continuous lines are corresponding theoretical results.

intensity-modulation through fiber dispersion was not included and the phase variation caused by the XPM process has a low-pass characteristic. In an ideal IMDD system, this phase modulation of the probe signal at the receiver does not affect the system performance. However, when a nonideal optical filter is involved, it may convert the phase noise to the intensity noise. This is significant in the low frequency part where XPM induced probe phase modulation is high. The discrepancy between theoretical and experimental results in the low frequency part of Fig. 2 is most likely caused by the frequency discrimination effect introduced through the narrow-band optical filter.

The XPM frequency response for a two-span system with 114 and $116 \mathrm{~km}$ of NZDSF is also measured and shown in Fig. 3 with 0.8 and $1.6 \mathrm{~nm}$ optical channel spaces. The optical power launched into each fiber span was $11.5 \mathrm{dBm}$. Corresponding theoretical results calculated from (11) are also 


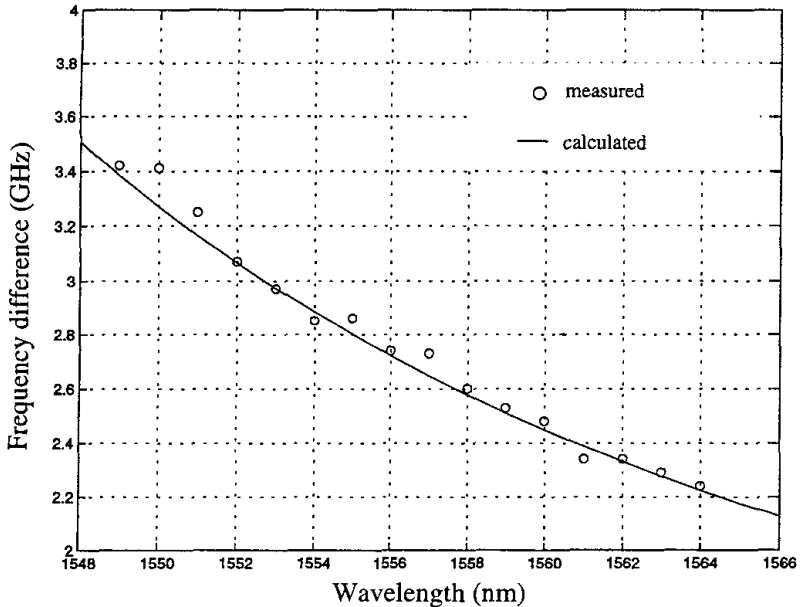

Fig. 4. Frequency differences between adjacent notches in the XPM spectrum versus signal wavelength with channel spacing $1.2 \mathrm{~nm}$. Calculation using fiber parameters $\lambda_{0}=1520.2 \mathrm{~nm}$, and $S_{0}=0.075 \mathrm{ps} / \mathrm{km} / \mathrm{nm}^{2}$.

displayed. Here, we see that the shape of XPM frequency response is strongly dependent on the channel spacing in a system with multiple optical spans. The ripples in the XPM spectral shown in Fig. 3 are due to interference between XPMinduced crosstalks which are created in different fiber spans. For this simple two-span system, it can be shown from (11) that the notches in the spectrum are located at frequencies which satisfy approximately the relation

$$
1+e^{i \Omega d_{j k} L_{1}}=0
$$

and, therefore, the frequency difference between adjacent notches of a spectrum is $\Delta f=1 /\left(d_{j k} L_{1}\right)$, where $L_{1}$ is the fiber length of the first span.

In order to verify the simple relationship shown in (12), we measured $\Delta f$ versus the signal wavelength at the fixed channel spacing of $1.2 \mathrm{~nm}$. The measured results are shown in Fig. 4. The theoretical results plotted in Fig. 4 are obtained using the fiber parameters listed above. This reasonably good agreement between theory and experiment suggests that this is also an alternative way to precisely measure fiber dispersion parameters in the system.

The XPM frequency response measured in a three-span system is shown in Fig. 5, where the first two spans are 114 and $116 \mathrm{~km}$ of NZDSF and the third span is $75 \mathrm{~km}$ of standard single-mode fiber (SMF). In this experiment, the EDFA's are adjusted such that the optical power launched into the first two spans of NZDSF is $11.5 \mathrm{dBm}$ and the power launched into the last span of standard SMF is $5 \mathrm{dBm}$. Taking into account that the spot size of the standard SMF is $8 \times 10^{-11} \mathrm{~m}^{2}$, which is larger than that of the dispersion shifted fiber, the XPM generated in the last span of standard SMF is significantly smaller than in the first two spans of NZDSF. Comparing Fig. 3 with Fig. 5 , the increase in the crosstalk power transfer function added by the last span of standard SMF is evident. The reason for this crosstalk increase is the high dispersion in the last span. This high dispersion results in a high efficiency of converting the phase modulation, created in the previous two

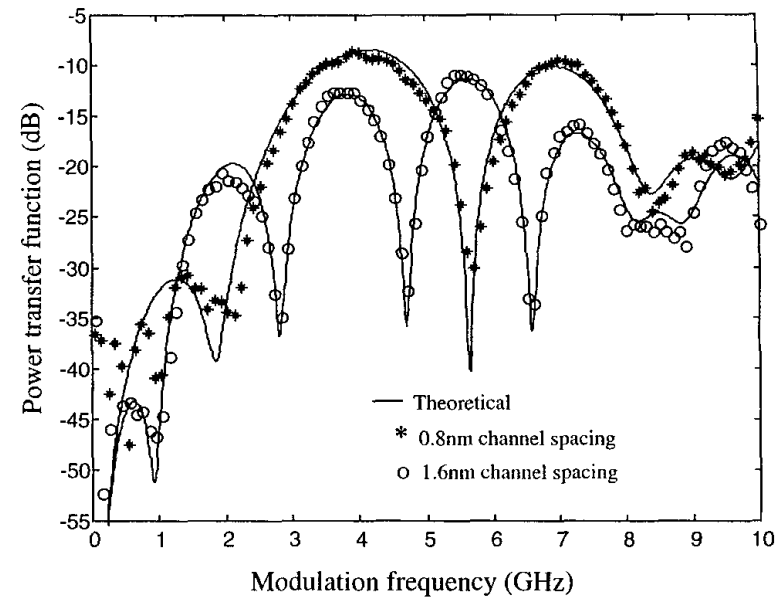

Fig. 5. XPM frequency response in the system with two spans (114 and 116 $\mathrm{km})$ of NZDSF and one span $(75 \mathrm{~km})$ of normal SMF. Stars: $0.8 \mathrm{~nm}$ channel spacing $\left(\lambda_{\text {probe }}=1559 \mathrm{~nm}\right.$ and $\left.\lambda_{\text {pump }}=1559.8 \mathrm{~nm}\right)$, open circles: $1.6 \mathrm{~nm}$ channel spacing $\left(\lambda_{\text {probe }}=1559 \mathrm{~nm}\right.$ and $\left.\lambda_{\text {pump }}=1560.6 \mathrm{~nm}\right)$. Continuous lines are corresponding theoretical results.

NZDSF spans, to intensity modulation. In other words, if the standard SMF was placed at the first span near the transmitter, the XPM crosstalk level would be much lower.

\section{SYSTEM IMPACT AND DISCUSSIONS}

So far, the normalized frequency response of XPM induced crosstalk has been analyzed. In this section we will discuss its impact on the performance of optical transmission systems. Even though the $\mathrm{CW}$ waveform of the probe channel used in our analysis simulates only the continuous " 1 "s in an nonreturn-to-zero (NRZ) bit pattern, the results may be generalized to pseudorandom signal waveforms. It is evident in (6) that the actual optical power fluctuation of the probe output caused by XPM is directly proportional to the unperturbed optical signal of the probe channel. Taking into account the real waveforms of both the pump and the probe, XPM induced crosstalk from the pump to the probe can be obtained as

$$
C_{j k}(t)=F^{-1}\left\{F\left[m_{k}(t)\right] \sqrt{\Delta p_{j k}(\Omega, L)} \sqrt{H_{j}(\Omega)}\right\} m_{j}(t)
$$

where $m_{j}(t)$ is the normalized probe waveform at the receiver and $m_{k}(t)$ is the normalized pump waveform at the transmitter. For pseudorandom bit patterns, $m_{j, k}(t)=u_{j, k}(t) / 2 P a v_{j, k}$ with $u_{j, k}$, the real waveforms, and $P a v_{j, k}$, the average optical powers. $F$ and $F^{-1}$ indicate Fourier and inverse Fourier transformations. $H_{j}(\Omega)$ is the receiver electrical power transfer function for the probe channel.

It is important to mention here that the expression of $\Delta p_{j k}(\Omega, L)$ was derived for a $\mathrm{CW}$ probe waveform, so (13) is not accurate during probe signal transitions between "0""s and "1"'s. In fact, XPM during probe signal transitions may introduce an additional time jitter, which is neglected in our analysis. However, a very recent work [9] verified experimentally that XPM-induced time jitter due to probe 


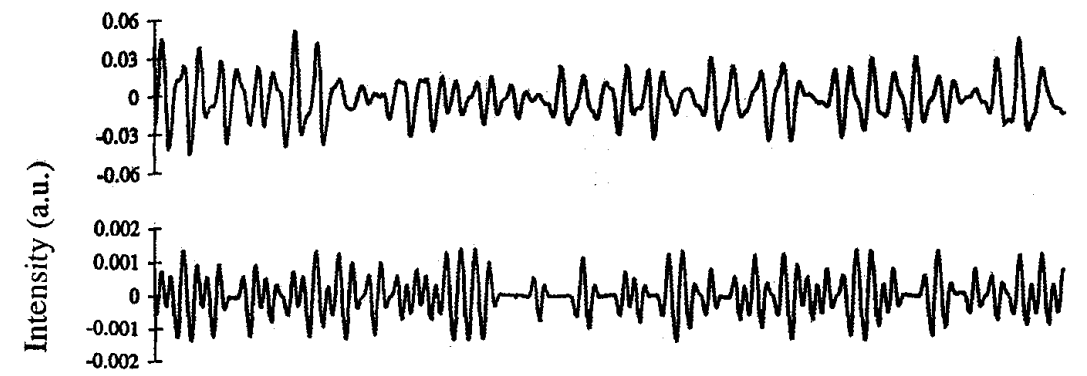

(a)

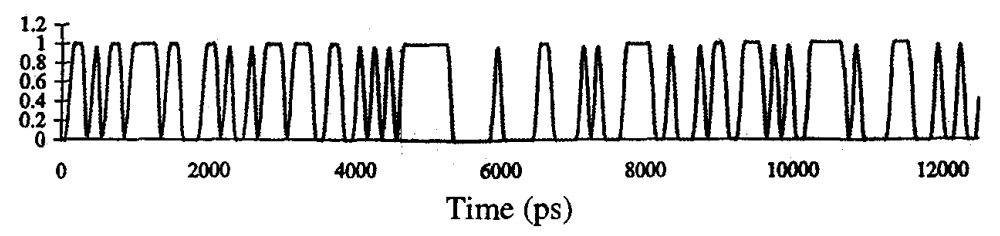

Fig. 6. Time domain waveforms. Trace (a): input pump signal [10 Gb/s $\left(2^{7}-1\right)$ pseudorandom bit pattern]. Trace (b): XPM crosstalk of the probe channel in a single span $130 \mathrm{~km}$ NZDSF system. Trace (c): XPM crosstalk of the probe channel in a three-span system with $130 \mathrm{~km}$ NZDSF +115 $\mathrm{km}$ NZDSF $+75 \mathrm{~km}$ normal SMF.

pattern effect is negligible compared to the XPM-induced eyeclosure at signal " 1 " and therefore, the CW probe method is an effective approach.

Another approximation in this analysis is the omission of pump waveform distortion during transmission. This may affect the details of the XPM crosstalk waveforms calculated by (13). However, the maximum amplitude of $C_{j, k}(t)$, which indicates the worst case system penalty, will not be affected as long as there is no significant change in the pump signal optical bandwidth during transmission.

In general, the impact of XPM crosstalk on the system performance depends on the bit rate of the pump channel, XPM power transfer function of the system as well as the baseband filter transfer function of the receiver.

\section{A. Waveforms of XPM Crosstalk}

In order to understand the impact of XPM on the system performance, it is helpful to look at time-domain waveforms involved in the XPM process. As an example, Fig. 6 trace (a) shows the normalized waveform (optical power) of the pump channel, which is a $10 \mathrm{~Gb} / \mathrm{s}\left(2^{7}-1\right)$ pseudorandom bit pattern, band-limited by a $7.5 \mathrm{GHz}$ raised-cosine filter. The probe was launched as a $\mathrm{CW}$ wave and its amplitude was normalized to "1." Due to XPM, the probe channel is intensity modulated by the pump and the waveforms created by the XPM process for two different system configurations are shown in Fig. 6. Trace (b) in Fig. 6 is obtained for a single span system with $130 \mathrm{~km}$ NZDSF, while trace (c) shows the XPM crosstalk waveform calculated for a three-span system with $130 \mathrm{~km} \mathrm{NZDSF}+$ $115 \mathrm{~km} \mathrm{NZDSF}+75 \mathrm{~km}$ standard SMF. Looking at these traces carefully, we can see that trace (b) clearly identifies a simple high-pass characteristic, which agrees with the similar waveform been recently measured and reported in [6] in a single span fiber system. However, in multispan systems, XPM transfer functions are more complicated. Trace (c) in Fig. 6 shows that the amplitude of the crosstalk associated with periodic " 0101 " pattern in the pump waveform is suppressed.

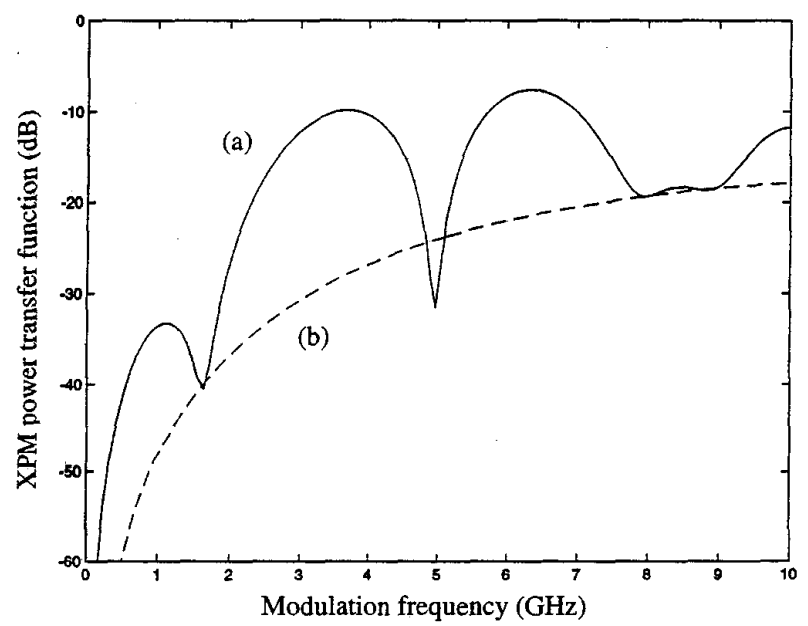

Fig. 7. XPM power transfer functions: (a) corresponds to trace (c) in Fig. 6 and (b) corresponds to trace (b) in Fig. 6.

In order to understand better the features in the timedomain waveforms obtained with different system configurations, Fig. 7 shows the XPM power transfer functions in the frequency-domain corresponding to the trace (b) and the trace (c) in Fig. 6. In the single span case, the crosstalk indeed has a simple high-pass characteristic. For the three-span system, however, the XPM power transfer function has a notch at the frequency close to the half bit rate, which suppresses the crosstalk of " 0101 " bit pattern in the time-domain.

It is worth mentioning that the crosstalk waveforms shown in Fig. 6 were calculated before an optical receiver. In reality, the transfer function and the frequency bandwidth of the receiver will reshape the crosstalk waveform and may have a strong impact in the system performance. After introducing a receiver transfer function, XPM induced eye closure "eyeclo" in the receiver of a system can be evaluated from the amplitude in the crosstalk waveform for the probe channel. The worst 


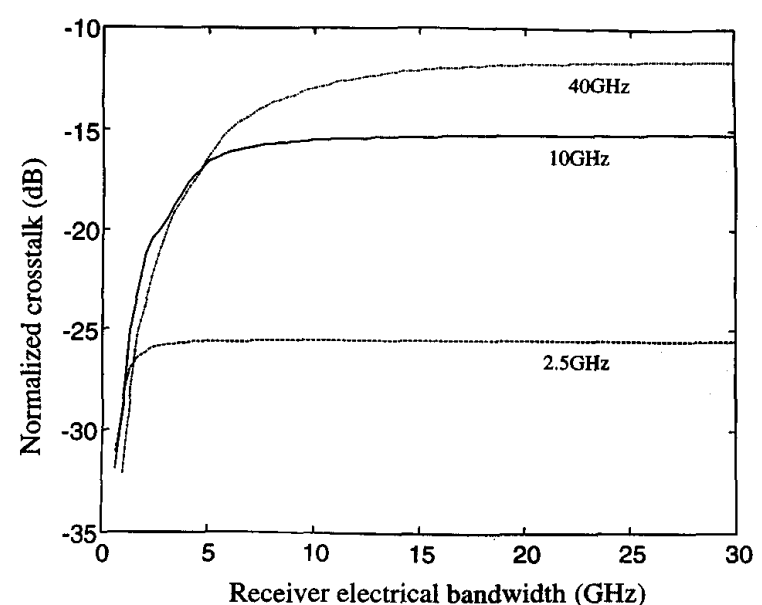

Fig. 8. Normalized power crosstalk levels versus the receiver bandwidth for $2.5,10$, and $40 \mathrm{~Gb} / \mathrm{s}$ bit rates in the pump channel. The $130-\mathrm{km}$ single-fiber span with fiber dispersion of $2.9 \mathrm{ps} / \mathrm{nm} / \mathrm{km}$ and optical channel spacing is 0.8 $\mathrm{nm}$. The launched pump optical power at each span is $11.5 \mathrm{dBm}$.

case eye closure happens with $m_{j}(t)=1$, and eyeclo ${ }_{\left(m_{j}=1\right)}=$ $\left\{\max \left[C_{j k}(t)\right]-\min \left[C_{j k}(t)\right]\right\} / 2$. We define this eye closure as normalized XPM crosstalk. In a complete system performance evaluation, this normalized XPM crosstalk penalty should be added on top of other penalties, such as those caused by dispersion and SPM. Considering the waveform distortion due to transmission impairments, the received probe waveform typically has $m_{j}(t) \leq 1$, especially for isolated " 1 "'s. Therefore, normalized XPM crosstalk gives a conservative measure of the system performance.

\section{B. Crosstalk Between Channels with Different Bit Rates}

In WDM optical networks, bit rates of different wavelength channels may not be the same. The impact of the probe channel bit rate on its sensitivity to XPM-induced crosstalk can be determined by the receiver bandwidth. Fig. 8 shows the normalized power crosstalk levels versus the receiver electrical bandwidth for $2.5,10$, and $40 \mathrm{~Gb} / \mathrm{s}$ bit rates in the pump channel. This figure was obtained for a single span system of $100 \mathrm{~km}$ with a dispersion of $2.9 \mathrm{ps} / \mathrm{nm} / \mathrm{km}$, launched optical power of $11.5 \mathrm{dBm}$ and a channel spacing of $0.8 \mathrm{~nm}$. In this particular system, we see that for the bit rate of higher than $10 \mathrm{~Gb} / \mathrm{s}$, the XPM-induced crosstalk is less sensitive to increases in the bitrate. This is because that the normalized XPM power transfer function peaks at approximately $15 \mathrm{GHz}$ for this system. When the pump spectrum is wider than 15 $\mathrm{GHz}$, the XPM crosstalk efficiency is greatly reduced. This is the reason why the difference in the XPM-induced crosstalk between 40 and $10 \mathrm{~Gb} / \mathrm{s}$ systems is much smaller than that between 10 and $2.5 \mathrm{~Gb} / \mathrm{s}$ systems.

Typical receiver bandwidths for $2.5,10$, and $40 \mathrm{~Gb} / \mathrm{s}$ systems are 1.75, 7.5, and $30 \mathrm{GHz}$, respectively. From Fig. 8, we can see that when the receiver bandwidth exceeds the bandwidth of the pump channel, the XPM-induced crosstalk level is no longer increased by increasing the receiver bandwidth. Indeed, the crosstalk between high bit-rate and low bit rate channels is comparable to the crosstalk between two low bit

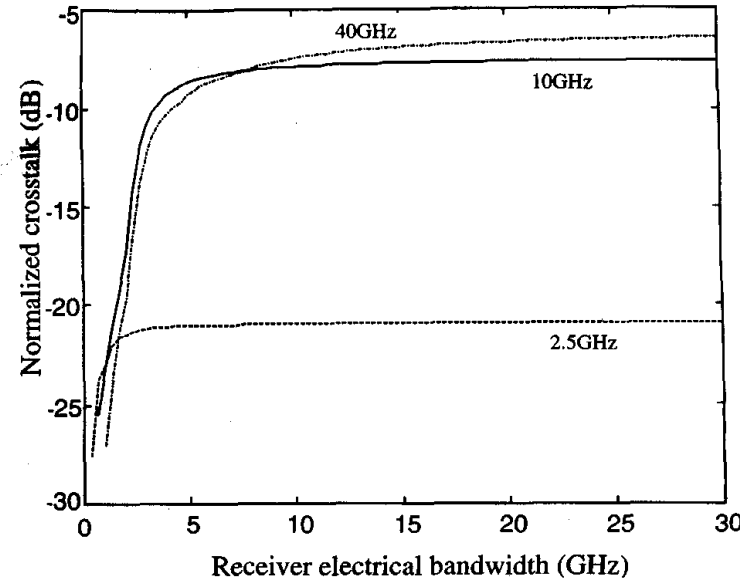

Fig. 9. Normalized power crosstalk levels versus the receiver bandwidth for $2.5,10$, and $40 \mathrm{~Gb} / \mathrm{s}$ bit rates in the pump channel. There are five cascaded fiber spans $(100 \mathrm{~km} / \mathrm{span})$ with fiber dispersion of $2.9 \mathrm{ps} / \mathrm{nm} / \mathrm{km}$ and optical channel spacing at $0.8 \mathrm{~nm}$. The launched pump optical power at each span is $8.5 \mathrm{dBm}$.

rate channels. An important implication of this is in hybrid WDM systems with different bit rate interleaving, for example, channels 1,3 , and 5 have high bitrates and channels 2,4 , and 6 have low bit rates. The XPM-induced crosstalk levels in both high and low bit rate channels are very similar and they are not higher than the crosstalk level in the system of low bit rate. However, when the channel spacing is too low, XPM crosstalk from channel 3 to channel 1 can be bigger than that from channel 2 with a low bitrate.

Similar to Fig. 8, Fig. 9 shows the normalized crosstalk levels versus receiver electrical bandwidth in a five-span NZDSF system with $100 \mathrm{~km} / \mathrm{span}$. The fiber dispersion is $2.9 \mathrm{ps} / \mathrm{nm} / \mathrm{km}$ and the launched optical power at each span is $8.5 \mathrm{dBm}$. Here, there is little difference in the crosstalk levels for the $10 \mathrm{~Gb} / \mathrm{s}$ system and the $40 \mathrm{~Gb} / \mathrm{s}$ system. This is because in systems with higher accumulated dispersion, the XPM power transfer function peaks at a lower frequency and the high-frequency components are strongly attenuated.

Fig. 10 shows the normalized crosstalk versus the fiber dispersion for the same system used to obtain Fig. 9. The fixed receiver bandwidths used for the 40,10 , and $2.5 \mathrm{~Gb} / \mathrm{s}$ systems are $30,7.5$, and $1.75 \mathrm{GHz}$, respectively. The worst case XPM crosstalk happens at lower dispersion with higher signal bitrate. It is worth noting that for the $10 \mathrm{~Gb} / \mathrm{s}$ system, the worst case XPM crosstalk happens when the fiber dispersion parameter is $2.5 \mathrm{ps} / \mathrm{nm} / \mathrm{km}$, and therefore the total accumulated dispersion of the system is $1250 \mathrm{ps} / \mathrm{nm}$, which is about the same as the dispersion limit for an uncompensated $10 \mathrm{~Gb} / \mathrm{s}$ system.

It needs to be pointed out that, for simplicity, in both Figs. 8 and 9 , the signal optical powers were chosen to be the same for systems with different bit rate. However, in practice, a higher power level is normally required for a system with a higher bit rate. A generalization of these results to the case with different signal power levels can be made using the simple linear dependence of XPM crosstalk on the launched power level as shown in (6). 


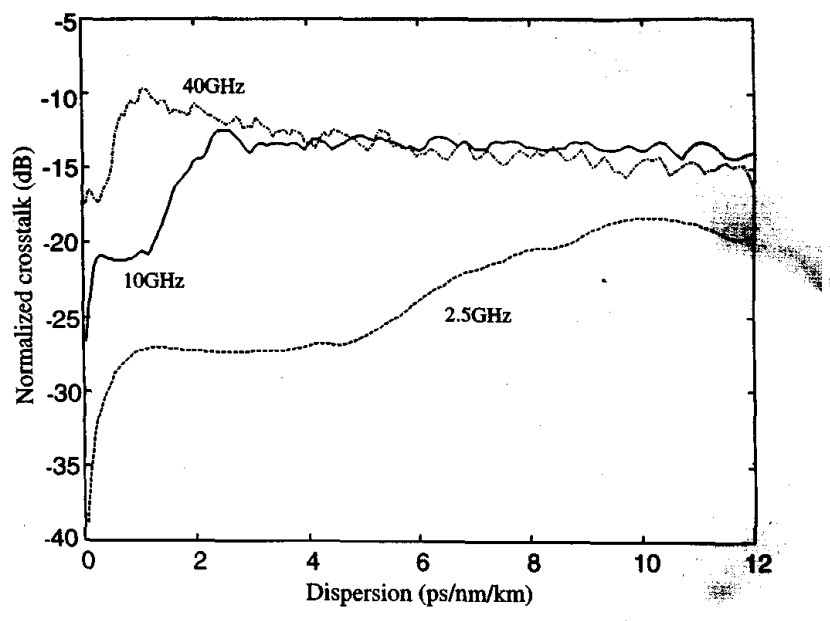

Fig. 10. Normalized power crosstalk levels versus the fiber dispersion for $2.5,10$, and $40 \mathrm{~Gb} / \mathrm{s}$ bit rates. There are five cascaded fiber spans (100 $\mathrm{km} / \mathrm{span}$ ) and the optical channel spacing is $0.8 \mathrm{~nm}$. The launched pump optical power at each span is $8.5 \mathrm{dBm}$.

Although most people would think that XPM crosstalk was significant only in low dispersion fibers, Fig. 10 clearly indicates that for uncompensated systems, before the system dispersion limit, higher dispersion generally produces more XPM crosstalk. On the other hand, in dispersion compensated optical systems, high local dispersion helps to reduce the XPM-induced phase modulation and low accumulated system dispersion will reduce the phase noise to intensity noise conversion.

\section{Dispersion Compensation Strategy}

It has been reported that XPM-induced crosstalk in fiber systems can be reduced by dispersion compensation [7]. Moreover, the position where the dispersion compensator is placed is also important. The least amount of dispersion compensation is required if the compensator is placed in front of the receiver. In this position, the dispersion compensator compensates XPM crosstalk created in all the fiber spans. The optimum amount of dispersion compensation for the purpose of XPM crosstalk reduction is about $50 \%$ of the total dispersion in the system [7]. Although this lumped compensation scheme requires the minimum amount of dispersion compensation, it does not give the best overall system performance.

Fig. 11 shows the normalized power crosstalk levels versus the percentage of dispersion compensation in a $10-\mathrm{Gb} / \mathrm{s}$, sixspan system with NZDSF of $100 \mathrm{~km} / \mathrm{span}$. The dispersion of transmission fiber is $2.9 \mathrm{ps} / \mathrm{nm} / \mathrm{km}$ and the launched optical power into each fiber span is $8.5 \mathrm{dBm}$. Nonlinear effects in the dispersion compensating fibers are neglected for simplicity. Different dispersion compensation schemes are compared in this figure. Trace (1) is obtained with compensation in each span. In this scheme XPM-induced crosstalk created from each span can be precisely compensated, so at $100 \%$ of compensation the XPM crosstalk is effectively reduced. Trace (2) was obtained with the dispersion compensator placed after every two spans. In this case, the value of dispersion compensation can only be optimized for either the first span

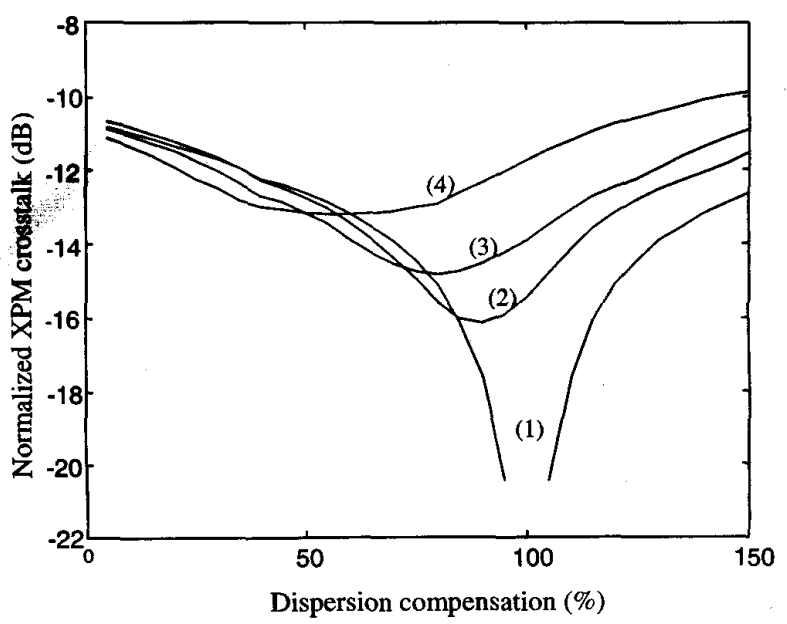

Fig. 11. Normalized power crosstalk levels versus the percentage of dispersion compensation in a $10-\mathrm{Gb} / \mathrm{s}$, six-span system $(100 \mathrm{~km} / \mathrm{span})$ with the fiber dispersion of $2.9 \mathrm{ps} / \mathrm{nm} / \mathrm{km}$. The $8.5 \mathrm{dBm}$ launched pump optical power at each fiber span: (1) dispersion compensation after each span, (2) dispersion compensation after every two spans, (3) dispersion compensation after every three spans, and (4) one lumped dispersion compensation in front of the receiver.

or the second span but not for both of them. The residual XPM crosstalk level is higher in this case than that with compensation in each span. Similarly, trace (3) in Fig. 11 was obtained with a dispersion compensator placed after every three spans and trace (4) is with only one lumped compensator placed in front of the receiver.

Obviously, when the number of dispersion compensators is reduced, the level of residual XPM crosstalk is higher and the optimum value of dispersion compensation is closer to $50 \%$ of the total system dispersion. Therefore, in systems where XPM-induced crosstalk is a significant impairment, per span dispersion compensation is recommended. However, this will increase the number of dispersion compensators and thus increase the cost.

\section{CONCLUSION}

We have investigated the spectral characteristics of XPM in multispan IMDD optical systems, both experimentally and theoretically. Interference between XPM-induced crosstalk components created in different amplified fiber spans has a strong impact on the overall frequency response of XPM crosstalk in the system. Reasonably good agreement between theory and experiment has been obtained.

In uncompensated optical systems, a decrease in fiber dispersion will increase XPM-induced phase modulation efficiency, while an increase in fiber dispersion will increase phase-to-intensity noise conversion efficiency. Dispersion compensation was shown to be an effective way to reduce XPM-induced crosstalk in IMDD systems. Different schemes of dispersion compensation in multispan optical fiber systems were evaluated and per span dispersion compensation was found to be the most effective way to minimize the effect of XPM crosstalk.

The crosstalk level between high and low bit rate channels was found to be similar to that between two low bit rate 
channels. This is due to the effect of the baseband filter in the optical receivers.

\section{ACKNOWLEDGMENT}

The authors are grateful to R. Butler of Sprint Corporation for his critical reading of the manuscript and two anonymous reviewers for their useful comments.

\section{REFERENCES}

[1] A. R. Chraplyvy, "Limitations of lightwave communications imposed by optical fiber nonlinearities," J. Lightwave Technol., vol. 8, pp. $1548-1557,1990$

[2] D. Marcuse, A. R. Chraplyvy, and R. W. Tkach, "Dependence of cross-phase modulation on channel number in fiber WDM systems," J. Lightwave Technol., vol. 12, pp. 885-890, 1994.

[3] J. Wang and K. Petermann, "Small signal analysis for dispersive optical fiber communication systems," J. Lightwave Technol., vol. 10, pp. 96-100, 1992.

[4] T.-K. Chiang, N. Kagi, M. E. Marhic, and L. Kazovsky, "Cross-phase modulation in fiber links with multiple optical amplifiers and dispersion compensators," J. Lightwave Technol., vol. 14, pp. 249-260, 1996.

[5] G. P. Agrawal, Nonlinear Fiber Optics. San Diego, CA: Academic, 1989.

[6] L. Rapp, "Experimental investigation of signal distortion induced by cross-phase modulation combined with distortion," IEEE Photon. Technol. Lett., vol. 9, pp. 1592-1594, Dec. 1997.

[7] R. A. Saunders, B. L. Patel, H. J. Harvey, and A. Robinson, "Impact of cross-phase modulation seeded modulation instability in $10 \mathrm{~Gb} / \mathrm{s}$ WDM systems and methods for its suppression," in Proc. Optic. Fiber Commun. Conf. OFC'97, Dallas, TX, Feb. 1997, paper WC4, p. 116.

[8] M. Shtaif and M. Eiselt, "Analysis of intensity interference caused by cross-phase modulation in dispersive optical fibers," IEEE Photon Technol. Lett., vol. 9, pp. 1592-1594, Dec. 1997.

[9] M. Eiselt, M. Shtaif, and L. D. Garrett, "Cross-phase modulation distortions in multi-span WDM systems," in Proc. Optic. Fiber Commun. Conf. OFC'99, San Diego, CA, Feb. 1999, paper ThC5.

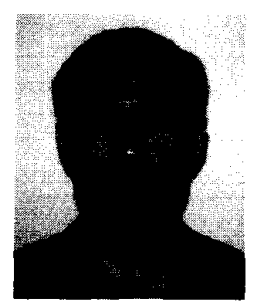

Rongqing Hui (SM'97) received the B.S. degree in microwave communications and the M.S. degree in lightwave technology from the Beijing University of Posts and Telecommunications, Beijing, China, in 1986 and 1988 , respectively. He received the Ph.D. degree in electrical engineering from the Politecnico di Torino, Torino, Italy, in 1993.

From 1982 to 1985 , he taught at the Physics department at Anhui University, Hefei, China, where he conducted research on optical fibers and fiber sensors. From 1985 to 1989 , he was with the optical Communication Laboratory, Beijing University of Posts and Telecommunications, where he worked in the field of coherent optical fiber communications systems and components. From 1989 to 1990, he held a research fellowship from the Fondazione Ugo Bordoni, Rome, Italy, working on nonlinear effects and optical injection locking of semiconductor laser devices. From 1990 to 1993, he was with the Department of Electronics, Politecnico di Torino, where he worked on optical communications and single-frequency semiconductor laser devices. During this period, he also held a fellowship with the Italian Telecommunications Research Center (CSELT), Torino, Italy, where he was working on polarization-insensitive coherent optical communication systems. He spent one year, from 1993 to 1994, as a Postdoctoral Research Fellow working on optical systems and networks architecture at the University of Ottawa, Ont., Canada. In 1994, he joined Bell-Northern Research (now part of Nortel), Ottawa, Canada, as a Member of Scientific Staff, where he has worked in the research and development of high-speed optical transport networks. Since September 1997, he has been a faculty member in the Department of Electrical Engineering and Computer Science, the University of Kansas, Lawrence. He has published more than 35 technical papers as an author or coauthor in leading engineering journals, in addition to numerous papers presented at international conferences. He also acted as a technical reviewer for various IEEE, Institution of Electrical Engineers (IEE), and Optical Society of America (OSA) journals.
Kenneth R. Demarest (S'78-M'79-SM'99) was born in Hackensack, NJ, on December 16, 1952. He received the B.S. degree in electrical engineering from John Brown University, Siloam Springs, AR, in 1974 and the M.S. and Ph.D. degrees in electrical engineering from the Ohio State University, Columbus, in 1976 and 1980 , respectively.

From 1974 to 1979 , he was a Graduate Research Associate with the ElectroScience Laboratory, Ohio State University. From 1979 to 1984 , he was an Assistant Professor in the electrical engineering department at Lafayette College, Easton, PA. Since 1984, he has been with the electrical engineering and computer science department at the University of Kansas, Lawrence, most recently as a Professor. His research interests are in the areas of fiber optic communications and electromagnetics. He is the author of a number of papers, book chapters, and the book Engineering Electromagnetics (Englewood Cliffs, NJ: Prentice Hall, 1998).

Dr. Demarest is a member of Eta Kappa $\mathrm{Nu}$ and the International Union of Radio Science Commission B.

Christopher T. Allen (M'94-SM'95), photograph and biography not available at the time of publication. 